\title{
BMJ Open Self-reported health status, treatment decision and survival in asymptomatic and symptomatic patients with aortic stenosis in a Western Norway population undergoing conservative treatment: a cross-sectional study with 18 months follow-up
} Kjersti Oterhals, ${ }^{1}$ Rune Haaverstad, ${ }^{1,2}$ Jan Erik Nordrehaug, ${ }^{2,3}$ Geir Egil Eide, ${ }^{4,5}$
Tone M Norekvăl

To cite: Oterhals K, Haaverstad R, Nordrehaug JE, et al. Self-reported health status, treatment decision and survival in asymptomatic and symptomatic patients with aortic stenosis in a Western Norway population undergoing conservative treatment: a cross-sectional study with 18 months follow-up. BMJ Open 2017;7:e016489. doi:10.1136/ bmjopen-2017-016489

- Prepublication history for this paper is available online. To view these files please visit the journal online (http://dx.doi. org/10.1136/bmjopen-2017016489).

Received 17 February 2017 Revised 5 July 2017 Accepted 19 July 2017

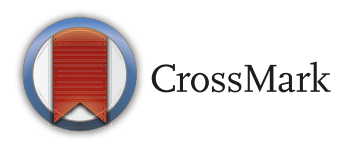

For numbered affiliations see end of article.

Correspondence to Dr Kjersti Oterhals; kjersti.oterhals@helse-bergen. no

\section{ABSTRACT}

Objectives To investigate symptoms and self-reported health of patients conservatively treated for aortic stenosis (AS) and to identify factors associated with treatment decision and patient outcomes.

Design A cross-sectional survey with an 18-month follow-up.

Setting One tertiary university hospital in Western Norway.

Participants In all, 1436 patients were diagnosed with AS between 2000 and 2012, and those 245 still under conservative treatment in 2013 were included in this study.

Primary and secondary outcome measures Primary outcome measures were symptoms and self-reported health status. Secondary outcomes were treatment decision and patient survival after 18 months.

Results A total of 136 patients with mean (SD) age 79 (12) years, $52 \%$ men responded. Among conservatively treated patients $77 \%$ were symptomatic. The symptom most frequently experienced was dyspnoea. Symptomatic patients reported worse physical and mental health compared with asymptomatic patients (effect size 1.24 and 0.74 , respectively). In addition, symptomatic patients reported significantly higher levels of anxiety and depression compared with asymptomatic patients. However, symptom status did not correlate with haemodynamic severity of AS. After 18 months, 117 (86\%) were still alive, 20\% had undergone surgical aortic valve replacement (AVR) and 7\% transcatheter aortic valve implantation (TAVI). When adjusting for age, gender, symptomatic status, severity of AS and European system for cardiac operative risk evaluation (EuroSCORE), patients with severe AS had more than sixfold chance of being scheduled for AVR or TAVI compared with those with moderate AS (HR 6.3, 95\% Cl 1.9 to 21.2, $p=0.003)$. Patients with EuroSCORE $\geq 11$ had less chance for undergoing AVR or TAVI compared with those with EuroSCORE $\leq 5$ (HR $0.06,95 \% \mathrm{Cl} 0.01$ to $0.46, \mathrm{p}=0.007$ ).
Conclusions Symptoms affected both physical and mental health in conservatively treated patients with AS. Many patients with symptomatic severe AS are not scheduled for surgery, despite the recommendations in current guidelines. The referral practice for AVR is a path for further investigation.

\section{INTRODUCTION}

Aortic stenosis (AS) is the most common heart valve disease in the Western world causing significant morbidity and mortality. As a result of an ageing population, the prevalence of AS is increasing. ${ }^{1}$ AS is most commonly caused by a degenerative calcification process leading to leaflet immobility, which in turn causes impaired blood flow through the heart and symptoms of pressure overload. ${ }^{2}$ The three cardinal symptoms

Strengths and limitations of this study

- The study is targeting an understudied group of patients as very few studies have investigated selfreported health in patients with aortic stenosis (AS) under conservative treatment.

- The study employs standardised and validated questionnaires.

- Patient-reported outcomes are important to inform health professionals as well as policymakers in order to improve the quality of care to patients with AS.

- Patients were diagnosed with AS 1-11 years before the survey.

- The study is limited by the moderate response rate, and that it was carried out as a single-centre study. 
of AS, indicating the need for clinical intervention, are breathlessness, chest pain and dizziness or syncope. ${ }^{1}$ An unknown proportion of patients remains asymptomatic for several years despite the presence of haemodynamically severe disease. ${ }^{3}$ Symptomatic AS has been associated with a sharp increase in death risk with an estimated $50 \%$ death rate at 2 years unless aortic valve replacement (AVR) is performed. ${ }^{3}$ While much is known about the pathophysiology of AS, little is known about the disease burden placed on patients' daily life, whether symptomatic or asymptomatic. ${ }^{4}$

Current European guidelines recommend AVR for patients with a class I assessment. These are patients who are symptomatic with severe AS, asymptomatic patients with severe systolic dysfunction or patients offered AVR as a concomitant procedure during another primary open cardiac surgery indication. ${ }^{5}$ Despite these recommendations, studies have documented poor adherence to evidence-based guidelines, as $33 \%-60 \%$ of the patients with severe symptomatic AS are inappropriately excluded from AVR. ${ }^{6}$ Hence, there seems to be a gap between what is recommended and the real clinical practice. For various reasons, there are a large percentage of suitable candidates that are currently not referred for AVR. ${ }^{5} 7$ Further, transcatheter aortic valve implantation (TAVI) has become widely accepted as an alternative to AVR and medical therapy for patients at high surgical risk. ${ }^{5}$

There are some reports on patient-reported outcomes in individuals with AS before and after AVR or TAVI. ${ }^{8}$ However, few studies have focused on the quality of life or self-reported health status of symptomatic or asymptomatic patients with AS who receive conservative treatment (ie, medical therapy) and in whom surgical intervention is postponed or declined either by the heart team or by the patient. ${ }^{49}$

The aims of this study were to investigate symptoms and self-reported health of patients conservatively treated for AS and to identify factors associated with treatment decision and patient outcomes.

\section{METHODS}

\section{Study design and participants}

A cross-sectional design was used to investigate factors related to patient-reported health status and the impact of valve disease on the patients' daily life. Patients were followed up for 18 months after the survey. Study endpoints were having undergone TAVI or AVR or all-cause death.

In April 2013, a postal questionnaire was sent along with a prestamped return envelope to 1436 patients $\geq 18$ years of age, able to write and understand Norwegian and diagnosed with AS in a tertiary university hospital in Western Norway. Results from the patients that had undergone AVR $(n=1191)$ are reported elsewhere. ${ }^{10}$ Two hundred and forty-five patients diagnosed with AS between 2000 and 2012 and still under conservative treatment were included in this study. To be included, candidate participants had to have echocardiographically verified AS in the native aortic valve, with at least a maximum transvalvular gradient of $\geq 40 \mathrm{~mm} \mathrm{Hg}$. Severe AS was defined according to current guidelines. ${ }^{5}$ Patients had to fulfil at least one of the following haemodynamic criteria: an aortic valve area (AVA) $<1 \mathrm{~cm}^{2}$; mean pressure gradient $>40 \mathrm{~mm} \mathrm{Hg}$ or peak aortic jet velocity $>4 \mathrm{~m} / \mathrm{s}$, as demonstrated by Doppler echocardiography. Moderate AS was defined as having an AVA of $1.5-1.0 \mathrm{~cm}^{2}$, a mean pressure gradient of $25-40 \mathrm{~mm} \mathrm{Hg}$ or a peak aortic jet velocity of $3.0-4.0 \mathrm{~m} / \mathrm{s}^{5}$

\section{Data collection}

Hospital information system registries and patient medical records were used to identify patients eligible for the study and to exclude patients with a maximum aortic gradient of less than $40 \mathrm{~mm} \mathrm{Hg}$, or patients who were cognitively impaired or deceased. Sociodemographic variables, smoking status, symptoms, comorbidities and physical and mental health status were obtained by means of patient self-reports. Clinical variables such as date of AS diagnosis, reasons for declining an AVR or TAVI, results from Doppler echocardiography examination regarding severity of AS and aortic regurgitation, treatment modalities, and survival were retrieved from patient medical records. Expected operative risk was calculated using numeric and logistic European system for cardiac operative risk evaluation (EuroSCORE) I classification (www. euroscore.org).

\section{Self-reported health status and symptoms}

Measurement of self-reported health status was obtained using the Short Form 12 (SF-12) health questionnaire. ${ }^{11}$ SF-12 has been used to assess health status in patients with AS undergoing AVR or TAVI. ${ }^{12}$ The SF-12 (standard V.1.0) questionnaire consists of 12 items. The first question asks the patient to rate his/her health as excellent, very good, good, fair or poor. In the survival analysis for the present study, the response categories 'excellent' and 'very good' and 'fair' and 'poor' were merged. SF-12 has two summary measures: a physical component summary (PCS) and a mental component summary (MCS). ${ }^{11}$ Each component summary results in a score ranging from 0 to 100. Summary scores are then standardised to a mean of 50 and a SD of 10. Higher scores represent better-perceived health status. Internal reliability (Cronbach's $\alpha$ ) was 0.89 and 0.88 for PSC and MSC, respectively.

To evaluate the burden of symptoms related to dyspnoea or heart failure, the Minnesota Living with Heart Failure Questionnaire (MLHFQ) was used. MLHFQ is a widely used disease-specific tool with well-documented validity, reliability and sensitivity for symptoms related to heart failure. ${ }^{13}{ }^{14}$ All of the symptoms listed on the MLHFQ are also symptoms that may occur in individuals with AS. ${ }^{15}$ Health impairment is evaluated using a six-point scale, ranging from 0 (no impact) to 5 (severe impact). The instrument produces a total score (21 items; range 0-105); a physical dimension subscore (PDS) (eight items; range 
0-40) and an emotional dimension subscore (EDS) (five items; range 0-25). Lower scores indicate better health. For MLHFQ, Cronbach's $\alpha$ was 0.94 for PDS and 0.88 for EDS.

The categories of symptomatic or asymptomatic AS were determined by patients' self-report in the survey. Symptoms of angina were obtained using a single question: "Have you had chest pain (yes/no)?" One question from the MLHFQ was used to determine the proportion of patients with dyspnoea: "Did your heart failure prevent you from living as you wanted during the last month by making you short of breath?" Possible answers ranged from 0 (no impact) to 5 (severe impact). Response values of $\geq 2$ were categorised as symptomatic. Dizziness/syncope was assessed by the question: "How much has dizziness/ syncope influenced your daily activities the last 4 weeks?” Possible responses were: 1 (not at all), 2 (a little), 3 (some), 4 (much) and 5 (very much). Response values of 3-5 were categorised as symptomatic. Cut-off points were set to avoid including patients who experienced very little discomfort as symptomatic. Timing of symptoms for valve replacement was not a goal in this cross-sectional study of symptomatic and asymptomatic patients with AS and their subsequent prognosis. Hence, patients were not assessed repeatedly for study purposes.

The Hospital Anxiety and Depression Scale (HADS) was used to assess possible symptoms of anxiety or depression. ${ }^{16}$ HADS consists of a seven-item subscale for anxiety (HADS-A) and a seven-item subscale for depression (HADS-D). For all items, responses are scored on a four-category scale, with 0 representing no symptoms and 3 representing maximum symptoms. The scores on each subscale range from 0 to 21 . For identifying possible cases of anxiety and depressive disorders, the HADS has an optimal cut-off score of at least 8 for both subscales. ${ }^{16}$ The Cronbach's $\alpha$ value was 0.86 for HADS-A and 0.75 for HADS-D.

The New York Heart Association (NYHA) Functional Classification was used to describe the impact of the disease on daily activities. ${ }^{17}$ NYHA classifies patients into four categories (I-IV), with higher classes indicating more severe symptoms and limitations in physical activity. The self-assessed NYHA classification tool asks patients to assign themselves to a NYHA class by ticking one of four boxes indicating categories that best describe their ability to perform physical activity. This tool is a well-documented and valid method of assessing symptoms of heart failure. $^{17}$

\section{Ethics}

The present study was conducted in accordance with the ethical guidelines contained in the World Medical Association's Declaration of Helsinki (2004) and was approved by the Regional Medical Ethics Committee of Western Norway (number 2010/01954). Information about the study, the possibility of withdrawing at any time and confidentiality issues were included in the letter that accompanied the questionnaire. Informed consent was taken as a patient returning the completed questionnaire. In accordance with the regional ethical committee, patients who failed to respond via mail were contacted once over telephone in order to encourage them to complete the questionnaire.

\section{Statistical analysis}

Descriptive statistics for continuous variables are presented as means and SDs. For comparisons between groups, the unpaired t-test was used. Descriptive statistics for categorical variables are presented as counts and proportions, and comparisons done using the exact $\chi^{2}$ test. Correlation between continuous variables was estimated by Pearson's correlation (r). Kaplan-Meier analysis was used to assess patient survival and cumulative incidence of AVR/TAVI after 18 months. Cox regression analysis was used to evaluate time-related events and their associations with baseline characteristics, such as age, gender, symptomatic status, severity of AS and EuroSCORE based on clinical experience and previous research. ${ }^{6}$ Results are reported as HRs and $95 \%$ CIs. No imputing of missing data was performed.

The statistical analyses were performed using SPSS for Windows V.22 (IBM), STATA/SE V.14.0 for Windows (February 2015), Matlab V.9.0 (The MathWorks, 2016) and Venn Diagram Plotter (http://omics.pnl.gov/software/venn-diagram-plotter). A two-sided $\mathrm{p}$ value $\leq 0.05$ was considered statistically significant. To evaluate the clinical importance of differences in self-reported physical and mental health of symptomatic and asymptomatic patients, we computed effect sizes (ESs statistics) by dividing the mean differences in scores by the SD of the norm data. ${ }^{18}$ To interpret the ES, we followed the suggestion of Cohen, and regarded ESs of $0.2-0.5$ as small, $0.5-0.8$ as moderate and 0.8 and above as large. ${ }^{18}$

\section{RESULTS}

\section{Patients' characteristics, symptoms and health status}

Of the 245 patients treated conservatively and not having undergone AVR or TAVI by April 2013, 137 patients $(56 \%)$ returned the questionnaire. One patient was excluded from further analysis due to a congenital subvalvular AS (figure 1). No statistically significant differences were found between responders and non-responders with respect to age $(\mathrm{p}=0.157)$ or gender $(\mathrm{p}=0.062)$.

The mean (SD) age was $79(12)$ years and $52 \%$ were men. One hundred and five $(77 \%)$ patients were symptomatic. The most frequently self-reported symptom was dyspnoea, 57 (71\%); followed by chest pain $49(61 \%)$ and dizziness/syncope 26 (33\%). Overlapping symptoms are shown in figure 2. Patients with symptomatic AS were older, had attained a lower educational level, were more often living alone, were placed in a higher NYHA class, had a higher EuroSCORE I and were more often on medication such as beta-blockers and statins, as compared with asymptomatic patients (table 1 ). 


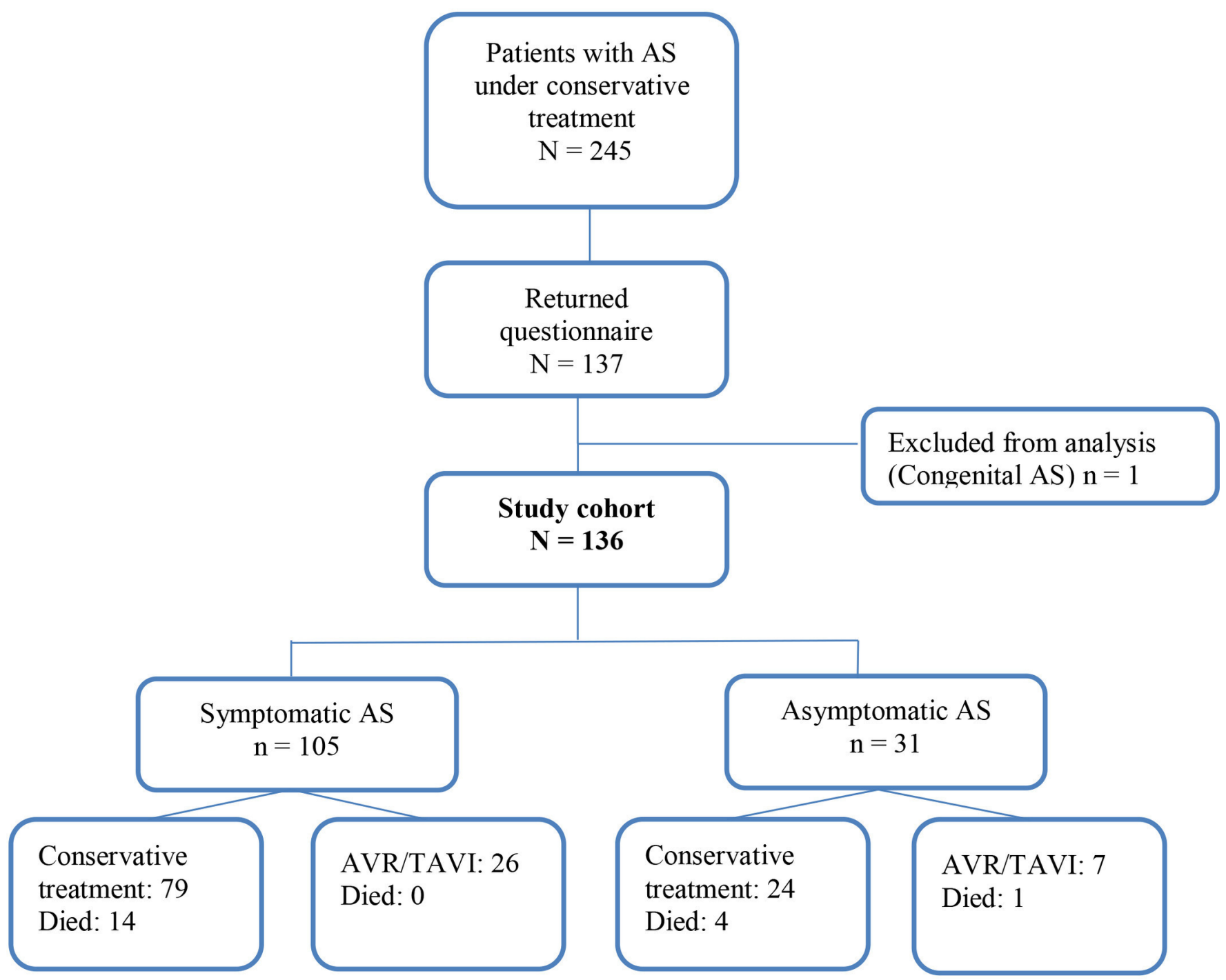

Figure 1 Flow chart showing patient with and without AVR participating in the study, and outcomes after 18 months followup for 136 patients aged 35 to 95 years under conservative treatment at the time of survey. Patients were diagnosed between the years 2000 and 2012 and were invited to complete the questionnaire in year 2013. AS, aortic stenosis; AVR, aortic valve replacement; TAVI, transcatheter aortic valve implantation.

Of the 136 patients, 22 (16\%) were not accepted for AVR by the heart team, while $12(9 \%)$ patients declined AVR by themselves. The remaining $102(75 \%)$ were considered as potential surgical candidates and remained under medical observation. The distribution of AS severity, symptomatic status and treatment decision at baseline are shown in figure 1 . No gender differences were found for severity of AS, chest pain, dyspnoea, dizziness/syncope or numbers of symptoms reported. Risk stratification of all 136 patients revealed that 29 patients $(21 \%)$ had a numeric EuroSCORE $\leq 5,81(60 \%)$ had a EuroSCORE between 6 and 10 and $26(19 \%)$ had a EuroSCORE of 11-15.

Patients with asymptomatic AS reported better physical and mental health status compared with symptomatic patients. The estimated ES for the differences in SF-12 measures between symptomatic and asymptomatic patients was 1.24 for the PCS and 0.74 for the MCS. The assessment of the impact of AS on the patients' daily life
(MLHFQ) showed that it had a significantly larger impact in symptomatic patients. In addition, asymptomatic patients had significantly lower HADS scores compared with symptomatic patients (table 2).

\section{Eighteen months follow-up}

Eighteen months after the survey, of the 136 patients, 117 $(86 \%)$ were still alive, Of whom $22(16 \%)$ had undergone isolated AVR, including 5 with AVR in combination with coronary artery bypass grafting and $10(7 \%)$ had undergone TAVI. Nineteen patients (14\%) had died; whereas 1 died 6 days after AVR. The flow chart of patient outcomes (survival and AVR/TAVI) within the 18 months follow-up is shown in figure 1.

Among the 102 individuals with medical observation at the time of the survey (figure 3), $22(21 \%)$ had undergone AVR, 7 (7\%) had TAVI and 9 (9\%) had died. Additionally, two patients were scheduled for AVR and two for TAVI during follow-up. Among the 22 patients 


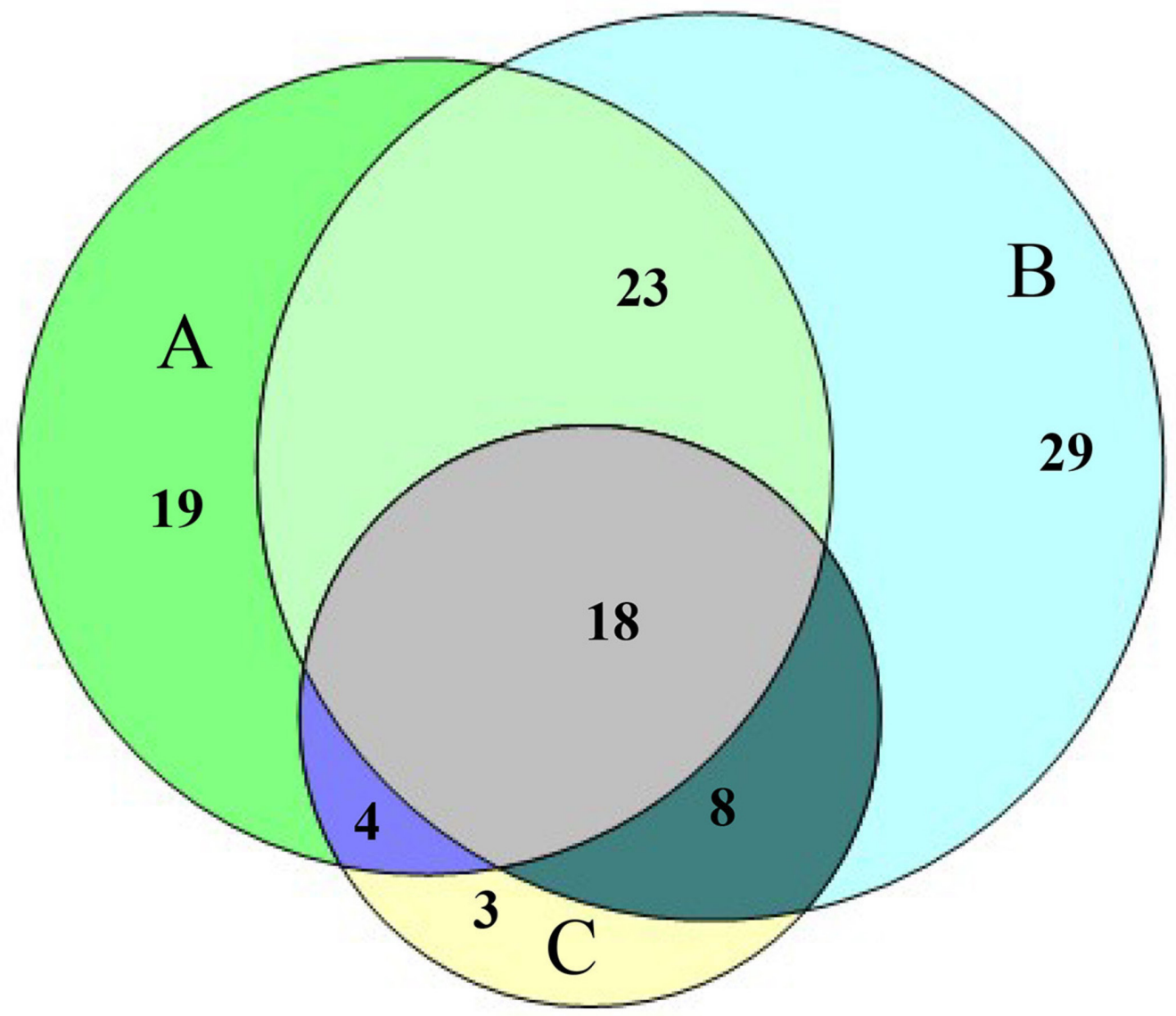

Figure 2 Number of symptomatic patients with aortic stenosis with overlapping symptoms ( $n=105)$. A, chest pain; B, dyspnoea; C, dizziness/syncope.

previously declined from AVR by the heart team, 3 (14\%) had undergone TAVI and $5(23 \%)$ had died. Four of the 12 patients who decided to receive conservative treatment had died and none had undergone AVR or TAVI.

Of the 20 patients with asymptomatic severe AS (table 1), only $3(15 \%)$ had undergone an exercise test. One of them had a rise in blood pressure and a decrease of $2 \mathrm{~mm}$ in the ST-segment on EKG, but no symptoms of angina. He was finally accepted for surgery, but died before the operation. In the other two cases, the patients experienced a slight rise in pulse, but in both cases the test ended prematurely due to limb fatigue.

After 18 months, of the 81 patients with symptomatic severe AS at baseline, 31 had still not undergone surgical treatment, and five had died prior to the end of the study. Of the latter, one had been accepted for AVR and one for TAVI. The remaining 26 patients were still treated conservatively after 18 months, either due to symptoms unrelated to AS $(n=3)$, patients' decision $(n=1)$, horizontal aortic root not eligible for TAVI $(\mathrm{n}=1)$, Alzheimer disease $(n=1)$ or vague symptoms $(n=3)$. The remaining 17 patients had, for unknown reasons, not been referred for cardiac surgery. Seven of the 20 patients with asymptomatic severe AS at baseline were treated with AVR $(n=5)$ or TAVI $(n=2)$ within 18 months. All of them developed symptoms, mainly dyspnoea before the intervention.
Multiple Cox regression analysis for selection of AVR or TAVI was performed and included age, gender, symptomatic status, severity of AS and EuroSCORE as variables (figure 4). Patients with severe AS had more than sixfold chance of being scheduled for AVR or TAVI compared with those with moderate AS (HR 6.3, 95\% CI 1.9 to 21.2, $p=0.003)$. EuroSCORE $\geq 11$ decreased the chance for undergoing AVR or TAVI compared with having EuroSCORE $\leq 5$ (HR $0.06,95 \%$ CI 0.01 to $0.458, p=0.007$ ).

Self-rated general health at baseline tended to predict event-free survival (figure 5A), and EuroSCORE tended to predict overall survival (figure $5 \mathrm{~B}$ ) by the 18 months follow-up, but the results were not statistically significant.

\section{DISCUSSION}

In the present study, the relationship between patient-reported outcomes and the severity of AS was investigated by employing well-established health status instruments. The results revealed that symptoms had a larger influence on the conservatively treated patients' physical and mental health than the severity of AS. AS severity alone, as measured by Doppler echocardiography examinations, did not differ between symptomatic and asymptomatic patients. 
Open Access

Table 1 Baseline characteristics of 136 patients aged 35 to 95 years diagnosed with severe-to-moderate aortic stenosis in the period 2000-2012 who replied to a postal questionnaire in 2013

\begin{tabular}{|c|c|c|c|c|}
\hline Variable & $\begin{array}{l}\text { All } \\
(n=136)\end{array}$ & $\begin{array}{l}\text { Symptomatic } \\
(n=105)\end{array}$ & Asymptomatic $(n=31)$ & p Value* \\
\hline \multicolumn{5}{|l|}{ Basic characteristics } \\
\hline Age in years; mean (SD) (range) & 79 (11) (35-95) & $80(10)$ & $75(12)$ & 0.025 \\
\hline Gender, men, n (\%) & $70(52)$ & $55(52)$ & $15(48)$ & 0.696 \\
\hline Living alone, n (\%) & $51(38)$ & $44(42)$ & $7(23)$ & 0.052 \\
\hline Education, n (\%) & & & & 0.037 \\
\hline Elementary school & $73(54)$ & $62(60)$ & $11(36)$ & \\
\hline High school & $33(24)$ & $23(22)$ & $10(32)$ & \\
\hline University/college & $29(22)$ & $19(18)$ & $10(32)$ & \\
\hline Smoking, n (\%) & & & & 0.121 \\
\hline Current smoker & $11(8)$ & $9(9)$ & $2(7)$ & \\
\hline Previous smoker & $58(43)$ & $49(47)$ & $9(30)$ & \\
\hline Never smoked & $66(49)$ & $47(45)$ & $19(63)$ & \\
\hline Sa-NYHA, mean (SD) & $2.18(0.87)$ & $2.34(0.88)$ & $1.66(0.55)$ & $<0.001$ \\
\hline NYHA I, n (\%) & $25(19)$ & $14(14)$ & $11(40)$ & \\
\hline NYHA II, n (\%) & $70(53)$ & $53(52)$ & $17(57)$ & \\
\hline NYHA III, n (\%) & $22(17)$ & $21(21)$ & $1(3)$ & \\
\hline NYHA IV, n (\%) & $14(11)$ & $14(14)$ & $0(0)$ & \\
\hline \multicolumn{5}{|c|}{ Doppler echocardiography examination $†$} \\
\hline Ejection fraction (\%), mean (SD) & $59(9)$ & $58(10)$ & $60(7)$ & 0.304 \\
\hline V-max (m/s), mean (SD) & $4.0(0.7)$ & $4.0(0.7)$ & $3.9(0.4)$ & 0.418 \\
\hline AVA $\left(\mathrm{cm}^{2} / \mathrm{BSA}\right)$, mean (SD) & $0.54(0.2)$ & $0.53(0.2)$ & $0.57(0.2)$ & 0.257 \\
\hline Mean aortic gradient, mean (SD) & $41(15)$ & $42(15)$ & $38(11)$ & 0.115 \\
\hline Severity of AS & & & & 0.159 \\
\hline Severe AS, n (\%) & $101(74)$ & $81(80) \ddagger$ & $20(20)^{\ddagger}$ & \\
\hline Moderate AS, n (\%) & $35(26)$ & $24(65) \ddagger$ & $11(35) \ddagger$ & \\
\hline Aortic regurgitation, n (\%) & & & & 0.067 \\
\hline Mild (1/4) & $55(40)$ & $43(41)$ & $19(18)$ & \\
\hline Moderate (2/4) & $27(20)$ & $19(18)$ & $8(25)$ & \\
\hline Moderate-to-severe (3/4) & $2(1.5)$ & $0(0)$ & $2(6)$ & \\
\hline EuroSCORE-log, mean (SD) & $12.8(11.6)$ & $13.9(11.8)$ & $9.0(10.3)$ & 0.027 \\
\hline EuroSCORE-numeric, mean (SD) & $8.0(3.1)$ & $8.3(3.1)$ & $6.9(2.8)$ & 0.022 \\
\hline Years since diagnosis, mean (SD) & $5.7(3.2)$ & $5.5(3.1)$ & $6.3(3.5)$ & 0.207 \\
\hline \multicolumn{5}{|l|}{ Medical history, n (\%) } \\
\hline Diuretics & $34(25)$ & $30(29)$ & $4(13)$ & 0.078 \\
\hline Beta-blockers & $87(64)$ & $71(68)$ & $16(52)$ & 0.104 \\
\hline Statins & $80(59)$ & $67(64)$ & $13(42)$ & 0.030 \\
\hline Myocardial infarction & $24(18)$ & $18(20)$ & $6(18)$ & 0.753 \\
\hline Stroke & $19(14)$ & $16(16)$ & $3(10)$ & 0.394 \\
\hline AF intermittent & $33(28)$ & $28(32)$ & $5(17)$ & 0.140 \\
\hline AF permanent & $8(7)$ & $7(7)$ & $1(3)$ & 0.454 \\
\hline COPD & $8(6)$ & $8(8)$ & $0(0)$ & 0.109 \\
\hline Arthritis & $25(19)$ & $22(22)$ & $3(10)$ & 0.139 \\
\hline Osteoporosis & $18(14)$ & $15(15)$ & $3(0)$ & 0.454 \\
\hline
\end{tabular}


Table 1 Continued

\begin{tabular}{|c|c|c|c|c|}
\hline Variable & $\begin{array}{l}\text { All } \\
(n=136)\end{array}$ & $\begin{array}{l}\text { Symptomatic } \\
(n=105)\end{array}$ & Asymptomatic $(n=31)$ & p Value* \\
\hline Cancer & $26(20)$ & $21(21)$ & $5(16)$ & 0.539 \\
\hline Previous CABG & $6(4)$ & $6(6)$ & $0(0)$ & 0.336 \\
\hline Pacemaker & $10(8)$ & $7(7)$ & $3(10)$ & 0.555 \\
\hline
\end{tabular}

${ }^{*}$ Tests comparing symptomatic versus asymptomatic (bold $p$ values denote significance at $p \leq 0.05$ ).

†Figures are mean values for moderate and severe stenosis.

†Symptomatic or asymptomatic patients are \% of all patients with severe or moderate AS.

AF, atrial fibrillation; AS, aortic stenosis; AVA, aortic valve area; BSA, body surface area; CABG, coronary artery bypass grafting; COPD, chronic obstructive pulmonary disease; EuroSCORE, European system for cardiac operative risk evaluation; NYHA, New York Heart Association; Sa-NYHA, self-assessed New York Heart Association functional classification; V-max, maximum jet velocity; PCl, percutanous coronary intervention.

Previous studies have shown that important outcomes such as symptoms, function and well-being are weakly associated with objective measures of disease severity. ${ }^{19}$ It is known that the degree of AS at the onset of symptoms differs among patients. ${ }^{3}$ The symptom most frequently experienced by patients in this study was dyspnoea. Dyspnoea was also the most frequent symptom observed in patients selected for AVR or TAVI. Since the presence of dyspnoea predicts worse survival for patients with $\mathrm{AS},{ }^{20}$ our treatment algorithm is well supported by clinical outcome studies ${ }^{20}$ and guidelines. ${ }^{5}$ In the present study, mild symptoms of shortness of breath were classified as asymptomatic AS. Surprisingly, nearly $60 \%$ of the asymptomatic patients classified themselves within NYHA class II, indicating they experienced shortness of breath or tiredness, or palpitations when performing strenuous activities. Rather than attributing these two symptoms solely to exercise induced AS another plausible explanation is the presence of other comorbidities, advanced age or their generally poor physical condition.

Symptomatic and asymptomatic patients also differed in physical and mental health status, with the latter having better scores. As the ES showed, this difference is also clinically relevant. ${ }^{18}$ Although neither the Doppler echocardiogram measurements (ie, AS severity) nor number of comorbidities differed between the two groups, the analyses showed that patient-reported outcomes did differ. This is in line with the reports of van Geldorp et al, who concluded that even minor AS symptoms may have a major impact on patients' physical and mental wellbeing, as well as quality of life. ${ }^{4}$ They also concluded that there was no relationship between stenosis severity and patients' physical or mental health, but symptoms severity according to NYHA classification corresponded well with the SF-36 scores.

Patients who graded their general health as fair or poor tended to have a lower 18-month event-free survival, as compared with those grading their health as good or better, but the difference was not statistically significant. Self-rated health has been shown to predict mortality 1 year after TAVI. ${ }^{21}$

In the present study, symptomatic patients reported significantly higher levels of both anxiety and depression compared with asymptomatic patients, indicating that AS symptoms have a great impact on mental health. Compared with the cohort of patients having undergone

Table 2 Baseline self-reported health status of 136 patients aged 35 to 95 years diagnosed with aortic stenosis in the period 2000-2012 responding to the questionnaire in 2013, data differentiated by symptomatic status

\begin{tabular}{lllcr}
\hline Variable & $\begin{array}{l}\text { All } \\
(\mathbf{n}=\mathbf{1 3 6})\end{array}$ & $\begin{array}{l}\text { Symptomatic } \\
(\mathbf{n}=\mathbf{1 0 5})\end{array}$ & $\begin{array}{l}\text { Asymptomatic } \\
(\mathbf{n = 3 1 )}\end{array}$ & $\mathbf{p}$ Value \\
\hline SF-12, PCS, mean (SD) & $36.8(11.7)$ & $33.8(11)$ & $46.2(9)$ & $<0.001$ \\
\hline SF-12, MCS, mean (SD) & $52.3(10.4)$ & $50.6(11)$ & $58.0(4)$ & $<0.001$ \\
\hline MLHFQ Physical mean (SD) & $11.8(11.3)$ & $14.9(11)$ & $3.3(5)$ & $<0.001$ \\
\hline MLHFQ Emotional mean (SD) & $3.1(5.1)$ & $3.9(6)$ & $0.6(2)$ & $<0.001$ \\
\hline HADS-A, mean (SD) & $3.9(3.4)$ & $4.2(4)$ & $2.9(2)$ & $\mathbf{0 . 0 3 6}$ \\
\hline HADS-D, mean (SD) & $4.5(3.4)$ & $5.0(4)$ & $2.7(2)$ & $<0.001$ \\
\hline HADS-A >8, n (\%) & $18(14)$ & $17(17)$ & $1(3)$ & 0.071 \\
\hline HADS-D >8, n (\%) & $24(18)$ & $22(22)$ & $2(7)$ & 0.056 \\
\hline
\end{tabular}

HADS-A, Hospital Anxiety and Depression Scale-anxiety component; HADS-D, Hospital Anxiety and Depression Scale-depression component; MCS, mental component summary of SF-12; MLHFQ, Minnesota Living with Heart Failure Questionnaire; PCS, physical component summary of SF-12; SF-12, Short Form 12. 


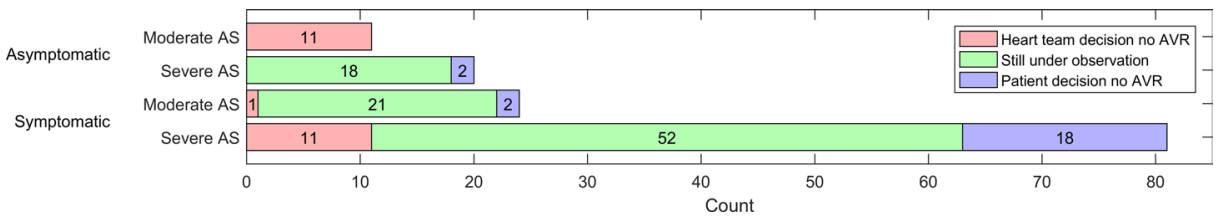

Figure 3 Reasons for not having undergone aortic valve replacement (AVR) at baseline in 136 patients with symptomatic and asymptomatic severe-to-moderate aortic stenosis (AS).

AVR responding to the same questionnaire, ${ }^{10}$ conservatively treated symptomatic patients in the present study reported a higher level of anxiety and depression. This supports the findings that AS symptoms have a negative impact on patients' mental health, suggesting that valve surgery can reduce this mental burden.

In symptomatic patients with severe AS, aortic valve surgery is generally recommended both by European and American guidelines. ${ }^{52}$ Fifty-five per cent of the patients who fulfilled the criteria for receiving AVR, either were not on the waiting list for AVR or had been treated with AVR or TAVI within 18 months after the survey. The low proportion of asymptomatic patient with AS who underwent exercise testing may suggest that the indication for surgery was underestimated. Symptomatic status can be difficult to determine, especially in elderly patients, as they tend to minimise or deny symptoms, or effectively reduce their physical activity level to avoid symptoms. ${ }^{23}$ One-third of the patients who report to be asymptomatic develop symptoms during exercise testing; thus, this type of testing is recommended to unmask symptoms in patients with severe AS. ${ }^{24}$ With appropriate supervision and monitoring, symptom-limited stress testing is safe in severe AS and can add important prognostic value especially in older people that might have problems performing a treadmill exercise test due to coexisting morbidities. ${ }^{24}$

A possible explanation why many patients are not referred for surgery despite severe symptomatic AS, is that the patients are discharged from the university hospital without being implemented into a treatment algorithm provided for the local hospitals, cardiologists in private practice or general practitioners. Multidisciplinary heart teams, together with the patients and their family, should conclude with either conservative treatment, AVR or TAVI according to current guidelines, shared decision-making, comorbidities and operative risks. Although the majority of the patients declined for surgery at baseline had a EuroSCORE of $>10$, a high EuroSCORE did not fully explain why some patients still were not referred for surgery. As EuroSCORE is known to overestimate the risk of postoperative mortality, especially in low-risk patients scheduled for isolated AVR, the postoperative mortality risk may have been overestimated in some of the patients. ${ }^{25}$

AS severity and not symptomatic status, predicted selection for surgical treatment in this cohort. The gap between the existing guidelines and their actual application has been demonstrated in previous studies. ${ }^{57}$ Close follow-up

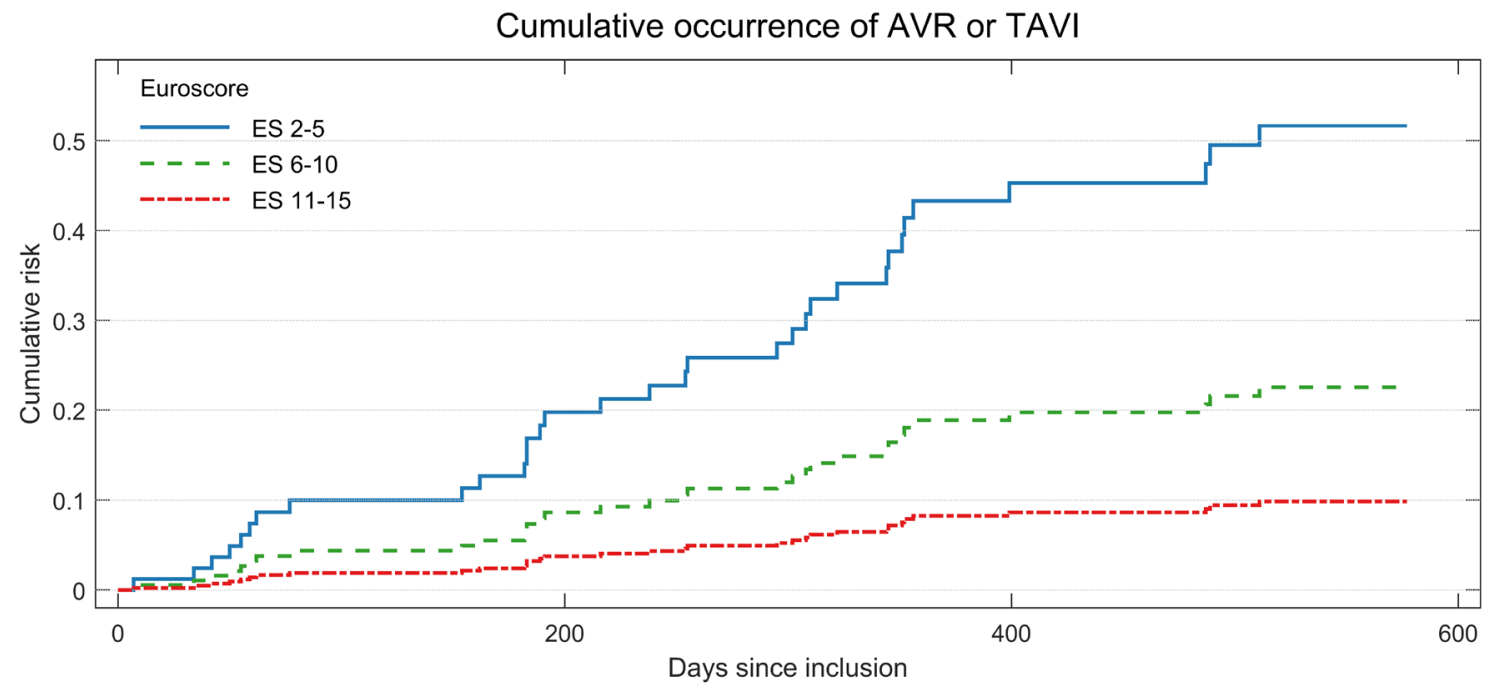

Figure 4 Cumulative occurrence of aortic valve replacement (AVR) or transcatheter aortic valve implantation (TAVI) from Cox model, according to European system for cardiac operative risk evaluation (EuroSCORE) range (likelihood ratio $p$ value (LR$p)=0.011)$ in patients with aortic stenosis within 18 months adjusted for gender ( $L R-p=0.336)$, age $(L R-p=0.223)$, symptomatic status $(L R-p=0.437)$ and severity of aortic stenosis $(L R-p=0.002)$. 
(A) Kaplan Meier: General health

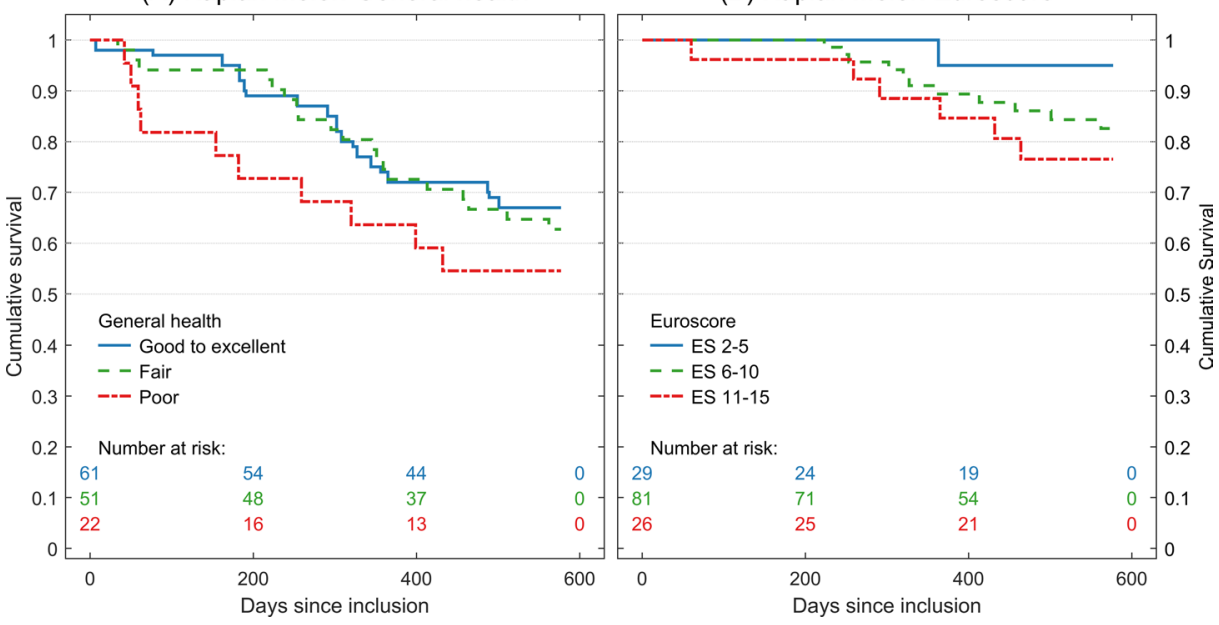

of the asymptomatic patients is also important, as severe symptoms or cardiac death may occur suddenly. ${ }^{26}$ There is robust evidence that AVR prolongs life in patients with symptomatic and severe AS. This is regardless of severity of symptoms or the response to medical treatment. ${ }^{3}$ It is also of utmost importance that health professionals inform patients with AS to contact their physician as soon as symptoms occur and close follow-up of asymptomatic patients is recommended. ${ }^{27}$ The optimal time for intervention is still open to debate. ${ }^{27}$ Some argue that early elective surgery in asymptomatic patients with severe AS might be worthwhile, since rapid deterioration is associated with the disease. ${ }^{26}$ Early surgery in patients with severe asymptomatic AS has also shown to improve long-term survival by decreasing cardiac mortality ${ }^{28}$ as well as lower 5-year incidences of all cause death and heart failure hospitalisation compared with conservative treated patients. ${ }^{29}$ In addition, an ongoing prospective multicentre randomised controlled trial (the AVATAR trail) is testing the hypothesis that elective AVR is superior to medical treatment until symptom onset in asymptomatic patients with isolated severe AVR and normal LVEF. ${ }^{30}$

\section{Study strengths and limitations}

Very few studies have investigated self-reported health in patients with AS under conservative treatment. Patient-reported outcomes are important to inform health professionals as well as policy-makers in order to improve the quality of care to patients with AS. Thus, the present study has some methodological limitations. It was carried out as a single-centre study, which may decrease the generalisability of the results. The sample, however, represents patients from both densely populated and rural areas. Another limitation is the retrospective design of the study and that we gathered only limited data prospectively. Further, a potential limitation is the moderate response rate of $56 \%$. A possible reason for this response rate can be that some of the patients were still asymptomatic, and perhaps not motivated to take the time to answer the questionnaire. Alternatively, patients with severe disease burden were incapable of completing the questionnaire.

\section{CONCLUSIONS}

Patients receiving conservative treatment for AS are an understudied group of patients with cardiac problems. The present study demonstrated that AS symptoms have great impact on patients' physical and mental health status. However, this impact does not reflect the severity of AS. Still, many patients with symptomatic severe AS are not scheduled for surgery, despite the recommendations in current guidelines. Our results indicate that the referral practice for aortic valve surgery ought to be carefully scrutinised. Further well-designed prospective studies are needed to fully understand the disease burden of AS and to optimise the timing of surgical intervention. Self-reported health status may be a valuable supplement to physical examination during the clinical evaluation of high-risk patients with AS.

\section{Author affiliations}

${ }^{1}$ Department of Heart Disease, Haukeland University Hospital, Bergen, Norway ${ }^{2}$ Department of Clinical Science, Faculty of Medicine and Dentistry, University of Bergen, Bergen, Norway

${ }^{3}$ Department of Cardiology, Stavanger University Hospital, Stavanger, Norway

${ }^{4}$ Department of Research and Development, Centre for Clinical Research, Haukeland University Hospital, Bergen, Norway

${ }^{5}$ Department of Global Public Health and Primary Care, University of Bergen, Bergen, Norway

Contributors $\mathrm{KO}, \mathrm{RH}, \mathrm{JEN}$ and TMN are responsible for study concept and design. $\mathrm{KO}$ and TMN are responsible for data collection. GEE, KO and TMN are responsible for data analysis. KO, RH, JEN and TMN are responsible for initial draft of manuscript. KO, RH, JEN, GEE and TMN are responsible for interpretation of data. All authors revised the paper critically for important intellectual content and approved the final manuscript. KO, GEE and TMN had full access to all of the data (including 
statistical reports and tables) in the study and take responsibility for the integrity of the data and the accuracy of the data analysis.

Funding This work was supported by a full research grant from the Western Norway Health Authority (grant number 911712) to KO. The study also received funding from The Norwegian Nursing Association (grant number 11/0104).

Competing interests None declared.

Ethics approval Regional medical ethics committee of Western Norway (number 2010/01954)

Provenance and peer review Not commissioned; externally peer reviewed.

Data sharing statement № additional data are available.

Open Access This is an Open Access article distributed in accordance with the Creative Commons Attribution Non Commercial (CC BY-NC 4.0) license, which permits others to distribute, remix, adapt, build upon this work non-commercially, and license their derivative works on different terms, provided the original work is properly cited and the use is non-commercial. See: http://creativecommons.org/ licenses/by-nc/4.0/

(C) Article author(s) (or their employer(s) unless otherwise stated in the text of the article) 2017. All rights reserved. No commercial use is permitted unless otherwise expressly granted.

\section{REFERENCES}

1. Rayner J, Coffey S, Newton J, et al. Aortic valve disease. Int J Clin Pract 2014;68:1209-15.

2. Freeman RV, Otto CM. Spectrum of calcific aortic valve disease: pathogenesis, disease progression, and treatment strategies. Circulation 2005;111:3316-26.

3. Otto CM, Prendergast B. Aortic-valve stenosis--from patients at risk to severe valve obstruction. N Engl J Med 2014;371:744-56.

4. van Geldorp MW, Heuvelman HJ, Kappetein AP, et al. Quality of life among patients with severe aortic stenosis. Neth Heart $J$ 2013;21:21-7.

5. Vahanian A, Alfieri O, Andreotti F, et al. Guidelines on the management of valvular heart disease (version 2012): the Joint Task Force on the Management of Valvular Heart Disease of the European Society of Cardiology (ESC) and the European Association for Cardio-Thoracic Surgery (EACTS). Eur J Cardiothorac Surg 2012;42:S1-44.

6. van Geldorp MW, van Gameren M, Kappetein AP, et al. Therapeutic decisions for patients with symptomatic severe aortic stenosis: room for improvement? Eur J Cardiothorac Surg 2009;35:953-7.

7. van Geldorp MW, Heuvelman HJ, Kappetein AP, et al. The effect of aortic valve replacement on quality of life in symptomatic patients with severe aortic stenosis. Neth Heart J 2013;21:28-35.

8. Shan L, Saxena A, McMahon R, et al. A systematic review on the quality of life benefits after aortic valve replacement in the elderly. $J$ Thorac Cardiovasc Surg 2013;145:1173-89.

9. Nugteren LB, Sandau KE. Critical review of health-related quality of life studies of patients with aortic stenosis. J Cardiovasc Nurs 2010;25:25-39.

10. Oterhals K, Hanssen TA, Haaverstad R, et al. Factors associated with poor self-reported health status after aortic valve replacement with or without concomitant bypass surgery. Eur J Cardiothorac Surg 2015;48:283-92.

11. Gandek B, Ware JE, Aaronson NK, et al. Cross-validation of item selection and scoring for the SF-12 Health Survey in nine countries: results from the IQOLA Project. International Quality of Life Assessment. J Clin Epidemiol 1998;51:1171-8.

12. Horrocks J, Closs J, Astin F. Quality of life in older adults with aortic stenosis: a narrative review. Int J Older People Nurs 2014;9.

13. Holmes C, Briffa N, . Patient-Reported Outcome Measures (PROMS) in patients undergoing heart valve surgery: why should we measure them and which instruments should we use? Open Heart 2016;3:e000315.

14. Supino PG, Borer JS, Franciosa JA, et al. Acceptability and psychometric properties of the Minnesota Living With Heart Failure Questionnaire among patients undergoing heart valve surgery: validation and comparison with SF-36. J Card Fail 2009;15:267-77.

15. Sandau KE, Boisjolie C, Hodges JS. Use of the Minnesota Living With Heart Failure Questionnaire among elderly patients with aortic stenosis: results from a pilot study. J Cardiovasc Nurs 2014;29:185-97.

16. Bjelland I, Dahl AA, Haug TT, et al. The validity of the Hospital Anxiety and Depression Scale. An updated literature review. J Psychosom Res 2002;52:69-77.

17. Holland R, Rechel B, Stepien K, et al. Patients' self-assessed functional status in heart failure by New York Heart Association class: a prognostic predictor of hospitalizations, quality of life and death. $J$ Card Fail 2010;16:150-6.

18. Fayers P, Machin D. Quality of life. The assessment, analysis and interpretation of patient-reported outcomes. Second edition. West Sussex, England: John Wiley \&Sons Ltd , 2007.

19. Sullivan M. The new subjective medicine: taking the patient's point of view on health care and health. Soc Sci Med 2003;56:1595-604.

20. Ross J, Braunwald E. Aortic Stenosis. Circulation 1968;38(1S5) :V-61--60.

21. Arnold SV, Spertus JA, Vemulapalli S, et al. Association of PatientReported Health Status With Long-Term Mortality After Transcatheter Aortic Valve Replacement: Report From the STS/ACC TVT Registry. Circ Cardiovasc Interv 2015;8:e002875.

22. Nishimura RA, Otto CM, Bonow RO, et al. 2014 AHA/ACC Guideline for the Management of Patients With Valvular Heart Disease: a report of the American College of Cardiology/American Heart Association Task Force on Practice Guidelines. Circulation 2014;129:e521-e643.

23. Henri $C$, Piérard LA, Lancellotti $P$, et al. Exercise testing and stress imaging in valvular heart disease. Can J Cardiol 2014;30:1012-26.

24. Rafique AM, Biner S, Ray I, et al. Meta-analysis of prognostic value of stress testing in patients with asymptomatic severe aortic stenosis. Am J Cardiol 2009;104:972-7.

25. Koene BM, van Straten AH, Soliman Hamad MA, et al. Predictive value of the additive and logistic EuroSCOREs in patients undergoing aortic valve replacement. J Cardiothorac Vasc Anesth 2011;25:1071-5.

26. Rosenhek R, Zilberszac R, Schemper M, et al. Natural history of very severe aortic stenosis. Circulation 2010;121:151-6.

27. Tastet L, Simard L, Clavel MA. Severe and Asymptomatic Aortic Stenosis Management Challenge: Knowing That We Do Not Really Know. Curr Treat Options Cardiovasc Med 2017;19:33.

28. Kang DH, Park SJ, Rim JH, et al. Early surgery versus conventional treatment in asymptomatic very severe aortic stenosis. Circulation 2010;121:1502-9.

29. Taniguchi T, Morimoto T, Shiomi H, et al. Initial Surgical Versus Conservative Strategies in Patients With Asymptomatic Severe Aortic Stenosis. J Am Coll Cardiol 2015;66:2827-38.

30. Banovic M, lung B, Bartunek J, et al. Rationale and design of the Aortic Valve replAcemenT versus conservative treatment in Asymptomatic seveRe aortic stenosis (AVATAR trial): A randomized multicenter controlled event-driven trial. Am Heart $J$ 2016;174:147-53. 